\title{
Vitamin A Deficiency Presenting with 'Itchy Eyes'
}

\author{
Matthew Hao Lee ${ }^{a}$ Marc G. Sarossy ${ }^{b, c}$ Ehud Zamir ${ }^{b, c}$ \\ ${ }^{a}$ Alfred Health, ${ }^{b}$ Centre for Eye Research Australia, The Royal Victorian Eye and Ear \\ Hospital, and ${ }^{C}$ Department of Ophthalmology, University of Melbourne, Melbourne, Vic., \\ Australia
}

\section{Key Words}

Nyctalopia $\cdot$ Bitot's spots $\cdot$ Xerophthalmia $\cdot$ Hypovitaminosis A $\cdot$ Electroretinogram

\begin{abstract}
We present the case of an 88-year-old female living in metropolitan Melbourne, Australia who developed vitamin A deficiency manifesting as 'itchy eyes' due to a bizarre dietary habit. Slit lamp examination revealed Bitot's spots and a subsequent vitamin A serum level test revealed severe deficiency. An electroretinogram showed grossly reduced a- and b-wave amplitudes consistent with generalised rod and cone dysfunction - these parameters showed marked improvement 5 months post supplementation. This case highlights the presence of vitamin A deficiency in the developed world and that a careful dietary history should be taken when assessing a patient complaining of 'itchy eyes'. Timely diagnosis and treatment may result in dramatic resolution of symptoms and signs as well as prevention of serious morbidity.

(C) 2015 The Author(s) Published by S. Karger AG, Basel
\end{abstract}

\section{Introduction}

Vitamin A deficiency is a leading cause of childhood blindness and mortality worldwide but is uncommon in developed countries. We report an unusual case of vitamin A deficiency in metropolitan Melbourne. 
Lee et al.: Vitamin A Deficiency Presenting with 'Itchy Eyes'

\section{Case Report}

An 88-year-old woman with a history of chronic lymphocytic leukaemia presented for routine ophthalmologic examination. She complained of 'itchy, burning eyes' and general visual decline. Her past ocular history included uneventful bilateral cataract surgery.

On examination, her initial Snellen visual acuity was $6 / 12$ on the right and $6 / 9$ on the left. Her intraocular pressure was $11 \mathrm{~mm} \mathrm{Hg}$ on the right and 10 on the left. Ishihara colour vision test was normal. Slit lamp examination of the eyes revealed frothy, dry-looking patches on the temporal bulbar conjunctiva bilaterally (fig. 1). The intraocular lenses were well placed bilaterally with slight posterior capsular opacification. The fundi were morphologically unremarkable.

The appearance of the ocular surface raised the suspicion of Bitot's spots from vitamin A deficiency. On further questioning, the patient reported 'food intolerance' for over 20 years. She had undergone extensive and unrevealing investigations but said she was intolerant to 'anything oily or fatty', and kept a strict diet limited to rice porridge, canned tuna and vitamin B supplements. A serum vitamin A level test was ordered, revealing vitamin A deficiency at a level of $0.1 \mu \mathrm{mol} / \mathrm{l}$ (normal: $1.6-2.3 \mu \mathrm{mol} / \mathrm{l}$ ). The patient was not aware of any problems with her night vision (nyctalopia).

Goldmann visual field testing showed full visual fields when tested with the larger III4e target but showed constriction to approximately $30^{\circ}$ on both sides when tested with the smaller Ie target (normal: $100^{\circ}$ ). This suggested a relative scotoma likely related to rod photoreceptor deficiency (fig. 2). An electroretinogram (ERG) was then performed; this test records the mass electrical activity from the retina when stimulated by various flash intensities in both dark-adapted (scotopic) and light-adapted (photopic) conditions. The scotopic rodspecific responses were grossly reduced whilst the cone ERGs (photopic, 30-Hz flicker) had less severely affected amplitudes but demonstrated increased latency (fig. 3). These findings, suggesting more severely affected rods than cones, are consistent with vitamin A deficiency.

Our patient was commenced on 100,000 IU of oral vitamin A supplements for 3 days, then 50,000 IU daily for 2 weeks $(50,000$ IU is equivalent to $15,000 \mu \mathrm{g}$ of vitamin A palmitate) $[1,2]$. She showed rapid resolution of symptoms and reported marked improvement in vision within $24 \mathrm{~h}$. A repeat ERG 5 months later (fig. 4) showed normalisation of scotopic responses and a mild improvement in photopic responses compared to the initial test.

\section{Discussion}

Vitamin A is an essential fat-soluble vitamin absorbed through the small intestine either as retinol (animal-derived) or carotene (plant- and vegetable-derived). Common food sources include milk, eggs and meat as well as dark leafy vegetables. It has a role in photoreceptor transduction of light to electrochemical energy as well as in the differentiation of epithelial cells on the ocular surface [3]. Vitamin A is stored in the liver, and the typical liver storage amount is sufficient for 2 years [4].

Vitamin A deficiency is a leading cause of childhood blindness and mortality worldwide, mostly in developing countries [5]. It is unusual in developed countries, where it may occur in the context of malnutrition (often related to alcoholism) or lipid malabsorption, as shown in table 1. Vitamin A deficiency is common after bariatric gastric bypass surgery, and improving awareness of this complication is of paramount importance in the ongoing medical management of this at-risk population [6]. 
Lee et al.: Vitamin A Deficiency Presenting with 'Itchy Eyes'

Xerophthalmia refers to the ocular manifestations associated with vitamin A deficiency; these include nyctalopia (night blindness), conjunctival and corneal xerosis (drying) and keratomalacia (corneal necrosis/ulceration) [5, 7]. Bitot's spots are patches of dry-looking conjunctiva with overlying frothy material which is composed of colonies of the diphtheroid Corynebacterium xerosis. This clinical finding is highly specific for vitamin A deficiency [8] and served as the presenting sign of a potentially sight-threatening condition in our patient. Nyctalopia is typically the first ophthalmic symptom and is a result of rod dysfunction, which happens earlier and to a greater extent than cone dysfunction [9]. An ERG can be useful for confirming and monitoring the retinopathy (fig. 3, fig. 4) - the main findings are reduced or undetectable rod ERGs with reduced amplitude cone ERGs of normal or delayed implicit time $[10,11]$. The relative resistance of cones can be explained by the fact that cones are more rapidly synthesised than rod pigments and hence may use up vitamin $A$ at the expense of rods [10]. Corneal surface signs are easily mistaken for epithelial erosions seen in keratoconjunctivitis sicca (dry eye syndrome) or exposure keratopathy [6], sometimes resulting in misdiagnosis. Advanced xerophthalmia manifests as keratomalacia, corneal infection and perforation.

The current treatment recommendation in Australia for patients with severe vitamin A deficiency is 100,000 IU of vitamin A daily for 3 days, followed by 50,000 IU daily for 2 weeks [1]. Intramuscular administration is reserved for patients with malabsorption or for those who are unable to tolerate oral medications. Managing the patient's risk factors is also essential. Typically, both nyctalopia and ocular surface symptoms improve within 1-5 days of vitamin A therapy [3].

The purpose of this review is to draw attention to the presence of nutritional deficiencies such as vitamin A deficiency in the developed world. Clinicians should be familiar with the aetiology and the subtle clinical manifestations of this disease in developed countries, especially in high-risk groups (table 1). Timely diagnosis and treatment may result in dramatic resolution of symptoms and signs as well as prevention of serious morbidity.

\section{Statement of Ethics}

The authors have no ethical conflicts to disclose.

\section{Disclosure Statement}

The authors have no conflicts of interest to disclose.

\section{References}

Product information: Vitamin A capsules. NSW, Phebra Pty Ltd, 2013, pp Product Information.

2 WHO/UNICEF/IVACG Task Force: Vitamin A Supplements: A Guide to Their Use in the Treatment and Prevention of Vitamin A Deficiency and Xerophthalmia, ed 2. Geneva, World Health Organization, 1997. http://whqlibdoc.who.int/publications/1997/9241545062.pdf?ua=1.

-3 Sommer A: Xerophthalmia and vitamin A status. Prog Retin Eye Res 1998;17:9-31.

4 Bellows L, Moore R: Fat-soluble vitamins: A, D, E and K: Fact Sheet, Colorado State University, 2012.

5 Al-Ani A, Ramaesh K, Wykes W: Diagnosis of vitamin A deficiency from its ocular manifestations in a British patient. Can J Ophthalmol 2009;44:e43.

-6 Lin P, Fintelmann RE, Khalifa YM, Bailony M, Jeng BH: Ocular surface disease secondary to vitamin A deficiency in the developed world: it still exists. Arch Ophthalmol 2011;129:798-804. 
Lee et al.: Vitamin A Deficiency Presenting with 'Itchy Eyes'

7 McLaughlin S, Welch J, MacDonald E, Mantry S, Ramaesh K: Xerophthalmia - a potential epidemic on our doorstep? Eye 2014;28:621-623.

8 Semba R: Handbook of Nutrition and Ophthalmology. Totowa, Humana Press Inc., 2010.

$>9$ Roncone DP: Xerophthalmia secondary to alcohol-induced malnutrition. Optometry 2006;77:124-133.

10 McBain VA, Egan CA, Pieris SJ, Supramaniam G, Webster AR, Bird AC, Holder GE: Functional observations in vitamin A deficiency: diagnosis and time course of recovery. Eye 2007;21:367-376.

11 Spits Y, De Laey JJ, Leroy BP: Rapid recovery of night blindness due to obesity surgery after vitamin A repletion therapy. Br J Ophthalmol 2004;88:583-585.

12 Sanli E, Figueira EC, Bhardwaj G, Watson SL, Francis IC: Tunnel vision and night blindness in a 52 -year-old man. Med J Aust 2011;195:287-288.

13 Soares-Mota M, Silva TA, Gomes LM, Pinto MAS, Mendonça LMC, Farias MLF, Nunes T, Ramalho A, Zaltman C: High prevalence of vitamin A deficiency in Crohn's disease patients according to serum retinol levels and the relative dose-response test. World J Gastroenterol 2015;21:1614-1620.

14 Braunstein A, Trief D, Wang NK, Chang S, Tsang SH: Vitamin A deficiency in New York City. Lancet 2010;376: 267.

15 Chae T, Foroozan R: Vitamin A deficiency in patients with a remote history of intestinal surgery. Br J Ophthalmol 2006;90:955-956.

16 Cooney TM, Johnson CS, Elner VM: Keratomalacia caused by psychiatric-induced dietary restrictions. Cornea 2007;26:995-997.

-17 Duignan E, Kenna P, Watson R, Fitzsimon S, Brosnahan D: Ophthalmic manifestations of vitamin A and D deficiency in two autistic teenagers: case reports and a review of the literature. Case Rep Ophthalmol 2015;6:24-29.

Table 1. Risk factors for vitamin A deficiency in the developed world

\begin{tabular}{|c|c|c|}
\hline Poor intake & Poor absorption & Poor metabolism/storage \\
\hline $\begin{array}{l}\text { Vegan/bizarre dietary practices } \\
{[12]}\end{array}$ & Crohn's disease [13] & $\begin{array}{l}\text { Liver disease including primary } \\
\text { biliary cirrhosis [6] and alcoholic } \\
\text { liver disease [7] }\end{array}$ \\
\hline
\end{tabular}

Anorexics [14] Intestinal/bariatric surgery [15]

Psychiatric patients [16] Pancreatic dysfunction including cystic fibrosis [14]

Autism [17] 
Case Reports in

Ophthalmology

\begin{tabular}{l|l|}
\hline Case Rep Ophthalmol 2015;6:427-434 \\
\hline DOI: 10.1159/000441969 & $\begin{array}{l}\text { @ } 2015 \text { The Author(s). Published by S. Karger AG, Basel } \\
\text { www.karger.com/cop }\end{array}$ \\
\hline
\end{tabular}

Lee et al.: Vitamin A Deficiency Presenting with 'Itchy Eyes'
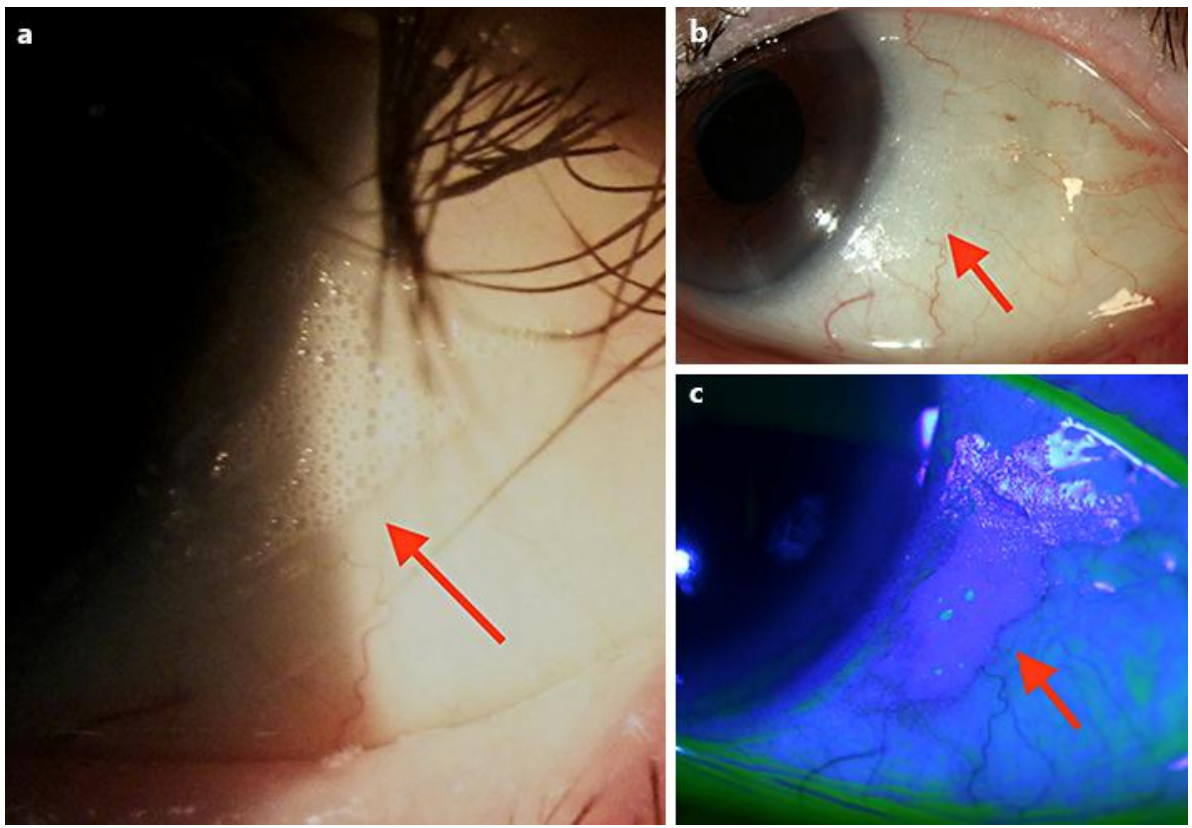

Fig. 1. Bitot's spot on the patient's temporal conjunctiva. Note frothy (a) or dry-looking (b) patches on the temporal bulbar conjunctiva. They are easier to see with fluorescein staining and blue light (c). 


\begin{tabular}{|c|c|c|}
\hline \multirow{3}{*}{$\begin{array}{r}\text { Case Reports in } \\
\text { Ophthalmology }\end{array}$} & \multirow{2}{*}{\multicolumn{2}{|c|}{ Case Rep Ophthalmol 2015;6:427-434 }} \\
\hline & & \\
\hline & DOI: $10.1159 / 000441969$ & $\begin{array}{l}\text { (c) } 2015 \text { The Author(s). Published by S. Karger AG, Basel } \\
\text { www.karger.com/cop }\end{array}$ \\
\hline
\end{tabular}
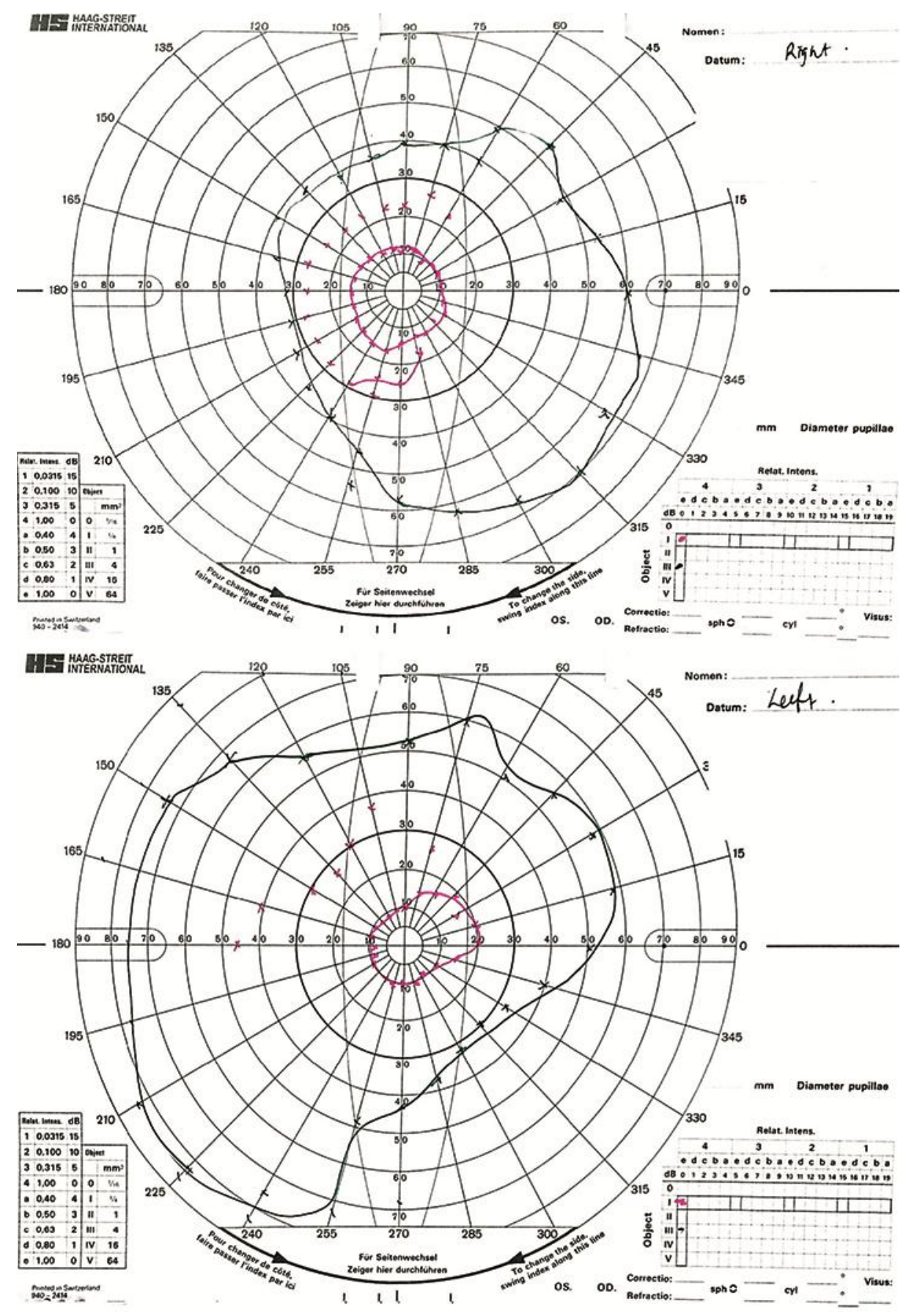

Fig. 2. Goldmann visual field analysis showed full visual fields when tested with the larger III4e target but showed constriction to approximately $30^{\circ}$ on both sides when tested with the smaller Ie target (normal: $100^{\circ}$ ). This suggested a relative scotoma and was likely related to rod photoreceptor deficiency. 
Lee et al.: Vitamin A Deficiency Presenting with 'Itchy Eyes'
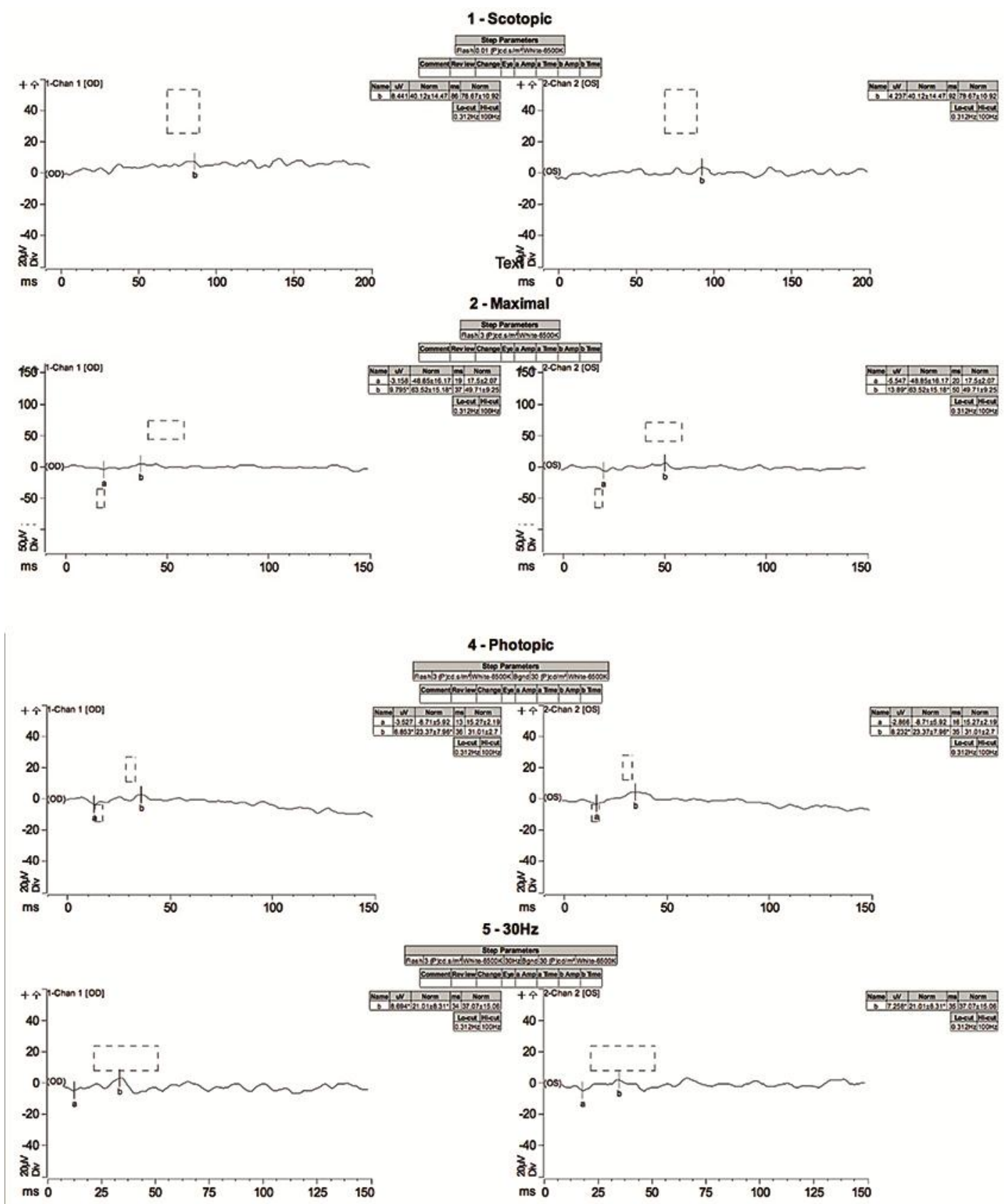

Fig. 3. Pretreatment ERG. Scotopic rod responses and the maximal or standard mixed rod-cone response were grossly subnormal. Photopic ERGs demonstrated borderline a-wave amplitudes, while b-waves had amplitudes that were approximately one-third of normal and had increased latencies. $30-\mathrm{Hz}$ flicker responses were half of normal bilaterally. 
Lee et al.: Vitamin A Deficiency Presenting with 'Itchy Eyes'
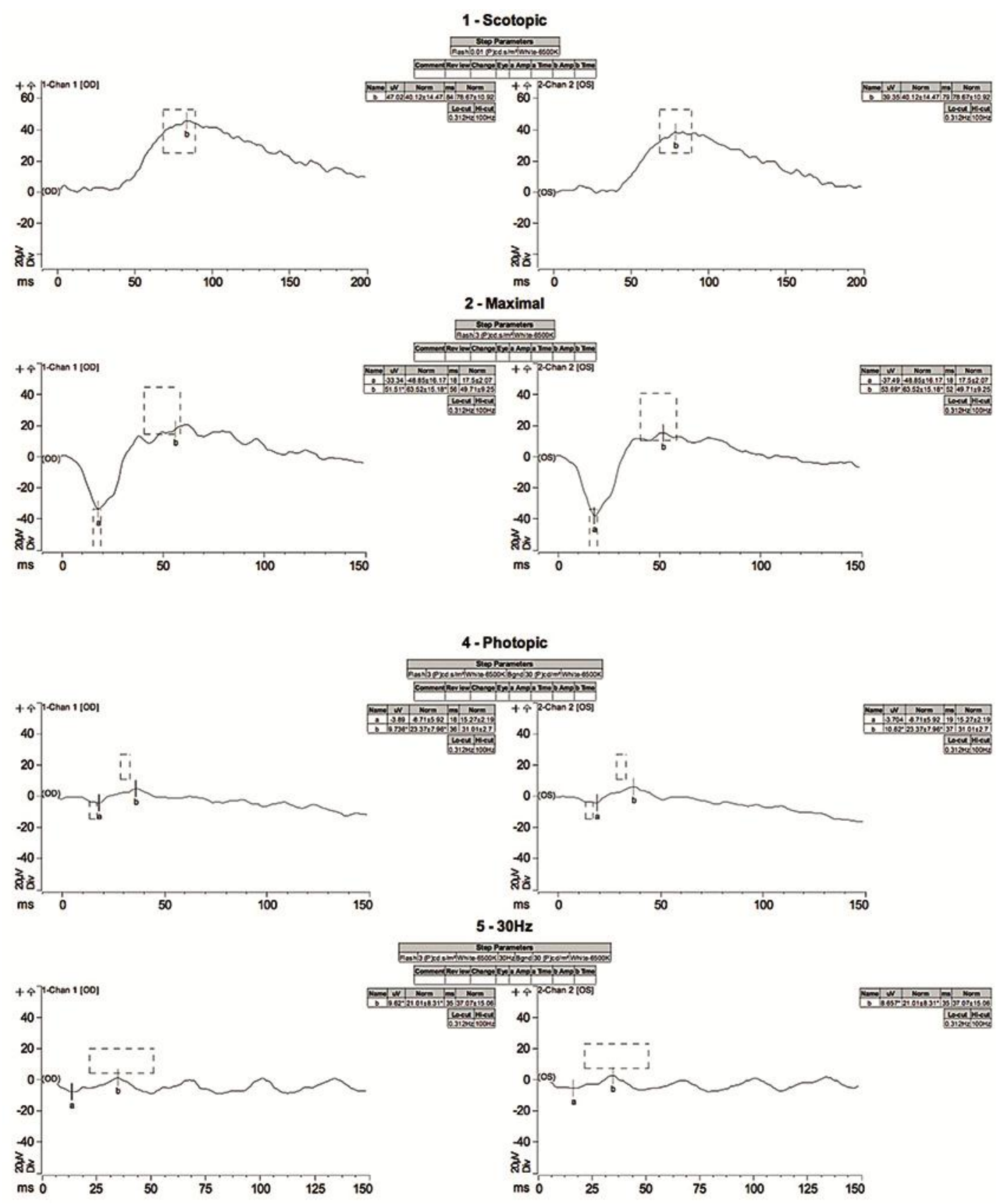

Fig. 4. Post-treatment ERG. Scotopic rod and standard responses showed normalisation of amplitudes after the standard course of vitamin A supplementation. The photopic response had improved amplitudes to about half of normal but no change in implicit time compared to initial testing. The $30-\mathrm{Hz}$ responses demonstrated marginal improvements in amplitudes. 